\title{
Clinical Characteristics and Prognosis of Elderly Small Cell Lung Cancer Patients Complicated with Hyponatremia: A Retrospective Analysis
}

\author{
YONGPING YANG ${ }^{1}$, NAIHONG SUN $^{2}$, PING SUN ${ }^{3}$ and LIANGMING ZHANG ${ }^{3}$ \\ ${ }^{1}$ Nursing Department, Yantai Yantaishan Hospital, Yantai, P.R. China; \\ ${ }^{2}$ Yantai Central Blood Station, Yantai, P.R. China; \\ ${ }^{3}$ Department of Oncology, Yantai Yuhuangding Hospital, \\ Affiliated with Medical College of Qingdao University, Yantai, P.R. China
}

\begin{abstract}
Aim: This study was designed to evaluate the clinical characteristics and prognosis of elderly small cell lung cancer (SCLC) patients complicated with hyponatremia, thus providing increased attention for appropriate intervention and improving outcomes in symptomatic subjects. Patients and Methods: The clinical data of 320 patients with SCLC in the Yuhuangding Hospital from March 1st, 2006, to March 1st, 2012, were studied retrospectively. The prognosis and possible association with hyponatremia was investigated. Results: The incidence rate of hyponatremia in SCLC was 46.56\% (149/320). The mean survival time was $1.10 \pm 0.42$ years in patients with normal values and $0.83 \pm 0.35$ years in patients with subnormal serum sodium. In the hyponatremia group, the mean survival time of corrected hyponatremia patients was $0.91 \pm 0.42$ years, which was significantly longer than uncorrected hyponatremia patients whose mean survival time was $0.68 \pm 0.26$ years $(t=2.75$, $p<0.05)$ after symptomatic treatment. The mean survival time of the normal group and the hyponatremia group in elderly patients had a tendency to decrease when compared to another group of patients younger than 60 years old. Hyponatremia at 1- and 3-year follow-up was associated with worse survival rates $(p<0.05)$. Conclusion: The severity of hyponatremia has unfavorable prognostic impacts. Elderly SCLC patients with hyponatremia are difficult to cure and associated with significantly shorter survival, especially in the uncorrected group. It is important that the cause of the
\end{abstract}

Correspondence to: Ping Sun and Liangming Zhang, Department of Oncology, Yantai Yuhuangding Hospital, 20 Yuhuangding East Road, Yantai, Shandong, P.R. China. Tel: +86 5356691999, Fax: +865356240341, e-mail: sunping20039@ hotmail.com

Key Words: Small cell lung cancer, elderly patients, hyponatremia, prognosis. hyponatremia is diagnosed at an early stage and precise medical treatment is provided.

Lung cancer remains the leading cause of cancer-related deaths worldwide, with small cell lung cancer (SCLC) being the most aggressive histologic subtype accounting for $15-20 \%$ of all cases (1-3). Despite comprehensive treatments, such as chemotherapy with or without radiation, the outcome of SCLC patients remains very poor, suggesting the need for identification of the major etiological factors involved.

As the geriatric population increases, the number of elderly SCLC patients continues to grow, with approximately half of limited-stage disease SCLC (LD-SCLC) patients now being more than 70 years old (4). It is, thus, important to be concerned about the combined modality therapy and specific care in such elderly patients (5).

Hyponatremia is the most common electrolyte abnormality due to the syndrome of inappropriate antidiuretic hormone hypersecretion (SIADH), a consequence of hypersecretion of vasopressin in SCLC patients $(6,7)$. It is associated with increased mortality, morbidity and length of hospital stay (8). Whether hyponatremia influences the survival of elderly SCLC patients or not remains controversial $(7,9-12)$. In the current study, we retrospectively analyzed if survival in elderly SCLC patients is impacted by hyponatremia and examined whether hyponatremia is a prognostic marker for SCLC. Our study also focused on early recognition and appropriate applied management that may significantly improve symptoms and prevent complications of hyponatremia.

\section{Patients and Methods}

Subjects and study design. A retrospective study was conducted. Consecutive patients first diagnosed with SCLC between March 1st, 2006, and March 1st, 2012, at the Yuhuangding Hospital Affiliated to Qingdao University were enrolled. Patients were classified to 4 groups in accordance with age and their serum 
sodium levels: patients 60 years old and older with normal level (Group A1), patients 60 years old and older with hyponatremia (Group A2), patients less than 60 years old with normal level (Group B1) and patients less than 60 years old with hyponatremia (Group B2). The patients' clinical information, including fundamental data, medical histories, routinely performed laboratory examinations and treatment modalities, was reviewed and summarized for analysis.

This study was approved by the Yuhuangding Hospital Ethics Committee. All patients provided their written informed consent.

Diagnostic criteria for hyponatremia. Normal serum sodium concentration $([\mathrm{Na}+])$ is between 135 and $145 \mathrm{mmol} / \mathrm{l}$. Hyponatremia is generally defined as a serum sodium concentration of $<135 \mathrm{mmol} / 1$. All hyponatremia patients are divided into the following subgroups: mild $(\geq 130 \mathrm{mmol} / \mathrm{l}$ and $<135 \mathrm{mmol} / \mathrm{l})$, moderate $(\geq 120 \mathrm{mmol} / \mathrm{l}$ and $<130 \mathrm{mmol} / \mathrm{l})$ and severe hyponatremia $(<120 \mathrm{mmol} / \mathrm{l})$.

Management of hyponatremia in SCLC patients. For etiological treatment of primary SCLC, chemotherapy is considered as the most appropriate choice. According to the National Comprehensive Cancer Network (NCCN) guidelines, the combination of cisplatin or carboplatin with etoposide is the first-line treatment. Cisplatin, as a platinum-based agent, however, has been associated with sideeffects, such as hepatotoxicity, nephrotoxicity and gastrointestinal reaction. If cancer progresses or relapses less than 6 months after the final dose of first-line treatment, therapy should be changed to second-line treatment, which is a regimen of a platinum-based agent plus irinotecan or cyclophosphamide, adriamycin (similar to epirubicin) and vincristine. The original regimen can be reused if cancer progresses 6 months after the final chemotherapy treatment. Radiotherapy is another important treatment in the management of SCLC. LD-SCLC is defined as a tumor confined to one hemi-thorax and regional lymph nodes and can be encompassed by a single radiation therapy port. These patients are given radical radiotherapy. They undergo thoracic radiotherapy with a dose of 54 Gy after 3 cycles of chemotherapy. Prophylactic cranial irradiation (PCI) is recommended for patients who achieve complete remission. On the other hand, palliative radiotherapy is given for extensive-stage disease SCLC (ED-SCLC). Local radiotherapy aiming at bone or brain metastases is adopted.

If etiological treatment cannot reduce inappropriate vasopressin secretion, specific therapy to correct serum sodium is necessary, i.e. fluid restriction or oral sodium substitution. However, we should also pay attention to the following issues: firstly, reasonable management between high-dose chemotherapy and restriction of fluid intake is important. Secondly, symptomatic and supportive treatment is required for hyponatremia patients with severe symptoms. These patients should restrict routine consumption of fluid to 500-800 $\mathrm{ml}$ a day. Meanwhile, administration of hypertonic saline solution is essential if patients experience extremely severe symptomatic hyponatremia. Hyponatremia should be corrected slowly in order to prevent the dreaded complication of osmotic demyelination syndrome (13).

The efficacy of the therapy should be evaluated after 2 curative cycles. If treatment is effective or stable, initial therapeutic regimens are continued until disease progression. No more than 6 systemic treatment cycles in standard intervals are recommended to induce remission.
Table I. Classification of patients in different groups.

\begin{tabular}{lccccc}
\hline Group & \multirow{2}{*}{$\begin{array}{c}\text { Number of } \\
\text { patients }\end{array}$} & $\begin{array}{c}\text { Normal } \\
\text { group }\end{array}$ & & \multicolumn{3}{c}{ Hyponatremia group } \\
\cline { 4 - 6 } & & & Mild & Moderate & Severe \\
\hline Group A & 222 & 120 & $55(53.92 \%)$ & $36(35.29 \%)$ & $11(10.78 \%)$ \\
Group B & 98 & 51 & $24(51.06 \%)$ & $19(40.43 \%)$ & $4(8.51 \%)$ \\
\hline
\end{tabular}

Statistical analysis. Statistical analysis was performed using the SPSS 22.0 statistical software package (IBM Corporation, Armonk, NY, USA). Comparison of the median survival time between the groups was presented as $(\chi \pm S)$ and performed using Student's $t$ tests. The method of Kaplan-Meier was used to calculate survival curves. To assess the statistical significance of differences between the crude survival curves, we performed log-rank tests. Multivariate testing with adjustments for age, gender, stage, response rates and performance status for patients in hyponatremia group and normal group was performed by Cox regression analyses. The level of statistical significance was defined as $p<0.05$.

\section{Results}

Comparison of the incidence of hyponatremia between two groups. Between March 1st, 2006, and March 1st, 2012, 320 patients in total, having an average age of $63.47 \pm 5.39$ years, were enrolled. The results showed that the total incidence of hyponatremia in our study was $46.56 \%$, which was generally identical with that reported in the literature. The incidence of Group A that contained 55 mild hyponatremia patients (53.92\%), 36 moderate (35.29\%) and 11 severe case patients $(10.78 \%)$ was $45.95 \%$. On the other hand, the incidence of Group B that included 24 mild case patients $(51.06 \%), 19$ moderate $(40.43 \%)$ and 4 severe cases $(8.51 \%)$ was $47.96 \%$ (Table I).

Comparison of the correction of hyponatremia between two groups. After treatment, 109 out of 149 (73.15\%) patients gained normal levels of serum sodium, while the rest of hyponatremia patients failed to achieve correction. The rate for uncorrected hyponatremia in Group A2 was 33.33\%, meanwhile the data in Group B2 revealed a rate of $12.77 \%$ $\left(\chi^{2}=2.83, p>0.05\right)$. Although the rate for uncorrected hyponatremia in the elderly group was obviously higher than control group, the difference was not statistically significant.

Correlation between hyponatremia and clinical characteristics. In total, 149 out of 320 patients $(46.56 \%)$ had serum sodium values below $135 \mathrm{mmol} / 1$. Hyponatremia was present in 85 out of $139(61.15 \%)$ patients with LDSCLC and 64 out of $181(35.36 \%)$ with ED-SCLC. The difference was statistically significant $(p<0.001)$. However, 
Table II. Correlation between hyponatremia and clinical characteristics.

\begin{tabular}{|c|c|c|c|c|c|}
\hline Characteristics & Total number $(\mathrm{n}=320)$ & Hyponatremia $(n=149)$ & Normal sodium $(\mathrm{n}=171)$ & Chi-square & $p$-Value \\
\hline \multicolumn{6}{|l|}{ Gender } \\
\hline Male & 219 & $96(43.84 \%)$ & $123(56.16 \%)$ & \multirow[t]{2}{*}{2.07} & \multirow[t]{2}{*}{0.15} \\
\hline Female & 101 & $53(52.48 \%)$ & $48(47.52 \%)$ & & \\
\hline \multicolumn{6}{|l|}{ Age } \\
\hline$<60$ years & 98 & $47(47.96 \%)$ & $51(52.04 \%)$ & \multirow[t]{2}{*}{0.11} & \multirow[t]{2}{*}{0.74} \\
\hline$\geq 60$ years & 222 & $102(45.95 \%)$ & $120(54.05 \%)$ & & \\
\hline \multicolumn{6}{|l|}{ Stage } \\
\hline LD & 139 & $85(61.15 \%)$ & $54(38.85 \%)$ & \multirow[t]{2}{*}{21.02} & \multirow[t]{2}{*}{$<0.001$} \\
\hline ED & 181 & $64(35.36 \%)$ & $117(64.64 \%)$ & & \\
\hline \multicolumn{6}{|l|}{ ECOG PS score } \\
\hline $0-1$ & 213 & $106(49.77 \%)$ & $107(50.23 \%)$ & \multirow[t]{2}{*}{2.63} & \multirow[t]{2}{*}{0.11} \\
\hline $2-3$ & 107 & $43(40.19 \%)$ & $64(59.81 \%)$ & & \\
\hline \multicolumn{6}{|l|}{ Response } \\
\hline $\mathrm{CR}+\mathrm{PR}+\mathrm{SD}$ & 142 & $67(47.18 \%)$ & $75(52.82 \%)$ & \multirow[t]{2}{*}{0.04} & \multirow[t]{2}{*}{0.84} \\
\hline PD & 178 & $82(46.07 \%)$ & $96(53.93 \%)$ & & \\
\hline
\end{tabular}

CR, Complete remission; PR, partial remission; SD, stable disease; PD, progressive disease; ECOG PS, Eastern Cooperative Oncology Group performance status; LD, limited-stage disease; ED, extensive-stage disease.

hyponatremia was not found to be associated with gender, age, Eastern Cooperative Oncology Group (ECOG) performance score and response (Table II).

Prognosis and median survival time. The mean survival time was $1.10 \pm 0.42$ years in the normal group and $0.83 \pm 0.35$ years in the hyponatremia group. There was a statistical difference between the two groups $(\mathrm{t}=3.28, p<0.05)$. In the hyponatremia group, the mean survival time of corrected hyponatremia patients was $0.91 \pm 0.42$ years, which was significantly longer than uncorrected hyponatremia patients whose mean survival time was $0.68 \pm 0.26$ years $(\mathrm{t}=2.75$, $p<0.05)$ after symptomatic treatment. The mean survival time of normal and hyponatremia group in Group A showed a decreasing tendency when compared with patients in Group B; nevertheless, there was no statistical difference $(p>0.05)$ (Table III).

Survival rate. The 1-year survival rate in the normal group was higher than the hyponatremia group, with the difference being statistically significant $\left(\chi^{2}=4.033, p=0.045\right)$. The survival rate in the corrected hyponatremia group was higher than the uncorrected group with statistical difference $\left(\chi^{2}=5.892, p=0.015\right)$.

The 3-year survival rate in the normal group was significantly higher than the hyponatremia group but the difference was not statistically significant $\left(\chi^{2}=2.066\right.$, $p=0.15)$. The survival rate in the corrected hyponatremia group was higher than the uncorrected group with a significant difference between the two groups $\left(\chi^{2}=4.786\right.$, $p=0.029$ ).
Table III. Median survival time in different groups.

\begin{tabular}{lllcc}
\hline Group & & $\mathrm{N}$ & $\begin{array}{c}\text { Median survival } \\
\text { time (years) }\end{array}$ \\
\hline A & A1 Normal & & 120 & $1.10 \pm 0.47$ \\
& A2 Hyponatremia & Corrected & 68 & $0.91 \pm 0.42$ \\
& & Uncorrected & 34 & $0.68 \pm 0.26$ \\
B & B1 Normal & & 51 & $1.23 \pm 0.61$ \\
& B2 Hyponatremia & Corrected & 41 & $1.02 \pm 0.77$ \\
& & Uncorrected & 6 & $0.75 \pm 0.53$ \\
\hline
\end{tabular}

The 5-year survival rate in the normal group was higher than the hyponatremia group; however, there was no statistically significant difference $\left(\chi^{2}=1.687, p=0.19\right)$ (Table IV).

Group A and group B were both divided into three subsets according to sodium values, respectively. Survival rates in the subsets are statistically significant both in group A and group B $(p<0.05)$ (Figures 1 and 2).

\section{Discussion}

Hyponatremia, resulting from SIADH, is one of the most frequent paraneoplastic syndromes in SCLC. As a subset of cancer, it has been revealed, in a large series of patients, that SIADH occurs in $15 \%$ of patients with SCLC (214 cases out of 1473 patients) compared to the incidence of $0.7 \%$ of patients with non-small cell lung cancer (NSCLC) (3 cases out of 427 patients) (14). Ectopically produced antidiuretic hormone $(\mathrm{ADH})$ by malignant tumors, drug-related 


\section{Survival rate in group $A$}
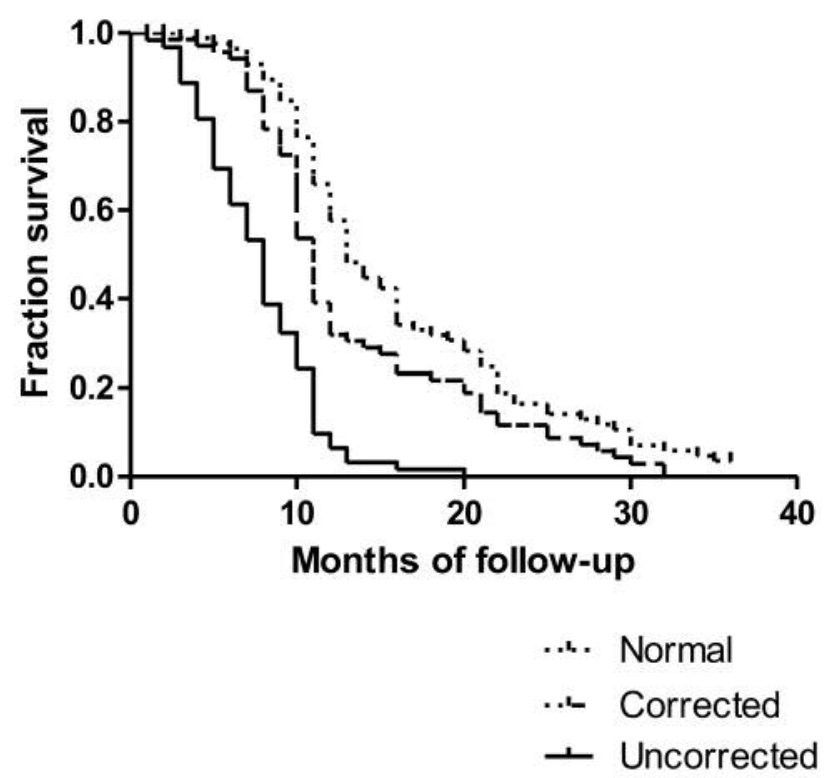

Figure 1. Survival rate in patients with normal sodium values $(n=120)$, corrected hyponatremia $(n=68)$ and uncorrected hyponatremia $(n=34)$ in group $A$ that contains patients aged 60 and older with normal sodium values and hyponatremia $(p<0.05)$.

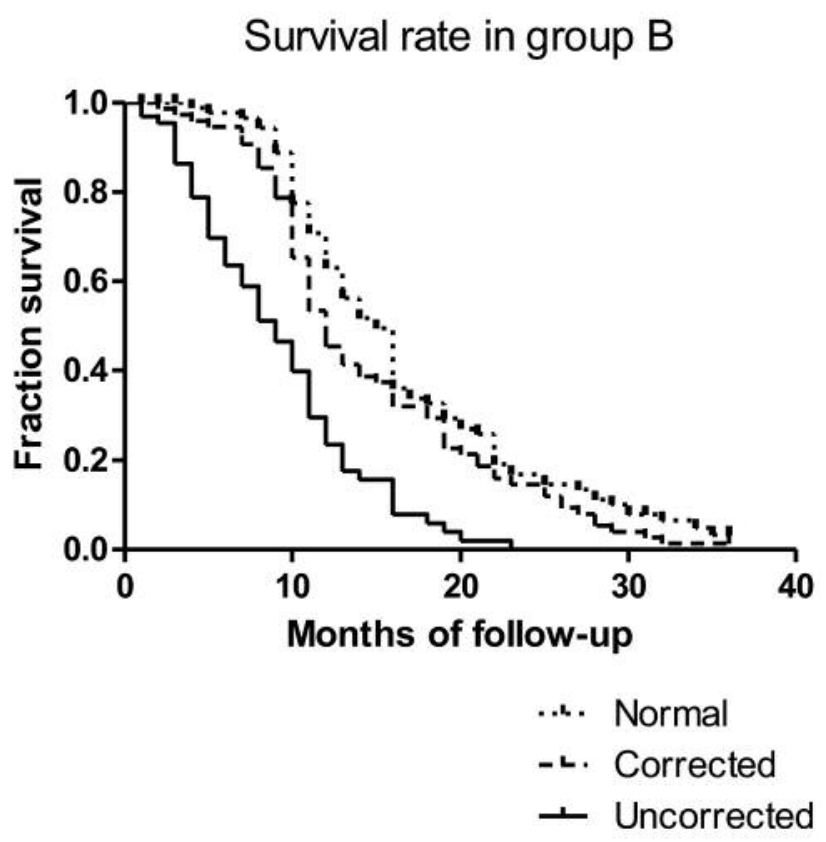

Figure 2. Survival rate in patients with normal sodium values $(n=51)$, corrected hyponatremia $(n=41)$ and uncorrected hyponatremia $(n=6)$ in group $B$ that contains patients less than 60 years old with normal sodium values and hyponatremia $(p<0.05)$.

Table IV. Survival rate in different groups.

\begin{tabular}{|c|c|c|c|c|c|}
\hline \multicolumn{2}{|c|}{ Group } & \multirow{2}{*}{$\begin{array}{c}\mathrm{N} \\
120\end{array}$} & \multirow{2}{*}{$\begin{array}{c}\begin{array}{c}\text { Survival cases } \\
(1-\text { year survival rate) }\end{array} \\
66(62.26 \%)\end{array}$} & \multirow{2}{*}{$\begin{array}{c}\begin{array}{c}\text { Survival cases } \\
\text { (3-year survival rate) }\end{array} \\
21(19.81 \%)\end{array}$} & \multirow{2}{*}{$\begin{array}{c}\begin{array}{c}\text { Survival cases } \\
(5-y e a r \text { survival rate })\end{array} \\
2(1.89 \%)\end{array}$} \\
\hline A & Normal & & & & \\
\hline & Hyponatremia & 102 & $34(30.36 \%)$ & $10(8.93 \%)$ & $0(0.00 \%)$ \\
\hline & Corrected & 68 & $30(38.46 \%)$ & $10(12.82 \%)$ & $0(0.00 \%)$ \\
\hline & Uncorrected & 34 & $4(11.76 \%)$ & $0(0.00 \%)$ & $0(0.00 \%)$ \\
\hline \multirow[t]{4}{*}{ B } & Normal & 51 & $46(70.77 \%)$ & $13(20.00 \%)$ & $3(4.61 \%)$ \\
\hline & Hyponatremia & 47 & $19(51.35 \%)$ & $5(13.51 \%)$ & $1(2.70 \%)$ \\
\hline & Corrected & 41 & $17(54.84 \%)$ & $5(16.13 \%)$ & $1(3.22 \%)$ \\
\hline & Uncorrected & 6 & $2(33.34 \%)$ & $0(0.00 \%)$ & $0(0.00 \%)$ \\
\hline
\end{tabular}

inductions (e.g. cisplatin), stress from surgery, central nervous system disorders and pain may cause SIADH in cancer patients $(15,16)$. However, it is neither easy nor practical to detect ADH levels in the patients' blood. Being usually asymptomatic, hyponatremia may cause clinical symptoms, such as nausea, fatigue, dizziness, headache, seizure and other neuropsychiatric symptoms (17). As reported in the literature, clinical significant hyponatremia is usually found before diagnosis of the malignancy (6). Special attention and careful management should be, thus, required because of neurological and life-threatening consequences.
In our study, $46.56 \%$ of patients showed a subnormal hyponatremia range of 120 to $135 \mathrm{mmol} / \mathrm{l}$ and $4.68 \%$ of patients exhibited severe hyponatremia defined as a value of $<120 \mathrm{mmol} / \mathrm{l}$. Our results were similar to those that have been well-documented. In 9 studies, including 1,684 patients with SCLC, summarized by Lassen et al., hyponatraemia $(<136 \mathrm{mmol} / \mathrm{l})$ was found in $24.6 \%$ of patients (18). In smaller groups, values from $25-44 \%$ were found $(9,19,20)$. The prognosis in the corrected hyponatremia group was significantly better than that of the uncorrected group. Whether hyponatremia is the direct cause of disease severity remains to be established. It has been suggested that hyponatremia has a 
remarkable influence on the prognosis of SCLC patients. Hyponatremia at 1- and 3-year follow-up was associated with worse survival rates but not at the 5-year follow-up. This could be due to the limited samples at the 5-year follow-up. Larger prospective studies should evaluate whether hyponatremia is a cause or a marker of poor prognosis.

It is important for SCLC patients with hyponatremia to correct their irregular sodium values effectively in order to improve disease prognosis. Hermes et al. reported that hyponatremia was a significant predictor for survival in patients with ED- and LD-SCLC after adjusting for known confounders, such as age, gender and performance status (17). Our study also showed that hyponatremia was correlated with ED and LD stage. However, in the current study, age, gender, response rate and performance status were not statistically different in patients with hyponatremia.

Correcting hyponatremia in SCLC patients may increase response to anticancer treatment and reduce morbidity and mortality. Therefore, effective treatment for hyponatremia is vitally important. Hypertonic saline and fluid restriction are recommended for conventional therapy. Availability of ADH receptor antagonists, such as tolvaptan, has been a major breakthrough in the management of hyponatremia as it may influence hyponatremia correction on cancer patients' outcomes (21).

High occurrence of hyponatremia in older patients was shown in our study. Among patients who were more than 60 years old, the incidence of hyponatremia was as high as $45.95 \%$ versus $47.96 \%$ in the comparison group, with this difference being significant. Hyponatremia in the elderly patients' group was harder to correct than in the younger group. The uncorrected rate of hyponatremia in the elderly patients' group reached $15.32 \%$, while that of the control group was $16.22 \%$. This situation could be due to medical conditions associated with elderly patients, such as hypertension, diabetes, stroke, renal failure and other comorbidities. Some of these conditions may also result in hyponatremia $(16,17)$. Elderly patients also showed poor tolerance of drugs, long disease course, reduced selfadjustment ability or other complications that, in fact, worsen prognosis. It, thus, becomes obvious that reasonable management might lead to a significant improvement of symptoms and quality of life in terms of clinical practice.

Our study has some limitations. First, the samples were from a single Hospital, with the population probably not representing a study cohort of general hospitalized cancer patients. Second, the study's retrospective nature could lead to selection bias. Elderly patients with severe or chronic courses might be investigated more often than patients with acute or mild courses. Last, a limited number of risk factors were examined. Management heterogeneity, physician's experience, an emerging and evolving evidence base and other clinical factors should also be evaluated and considered for their influence on outcomes in future studies.

\section{Conflicts of Interest}

The Authors declare that there are no conflicts of interest regarding the publication of this paper.

\section{Acknowledgements}

The Authors would like to express our sincere gratitude to the patients and their families for agreeing to participate in this work. This work has been supported by Science and Technology Development Foundation of Yantai (No.2014WS056).

\section{References}

1 Van Meerbeeck JP, Fennell DA and De Ruysscher DK: Small cell lung cancer. Lancet 378(9804): 1741-1755, 2011.

2 Gridelli C, Langer C, Maione P, Rossi A and Schild SE: Lung cancer in the elderly. J Clin Oncol 25(14): 1898-1907, 2007.

3 Govindan R, Page N, Morgenstern D, Read W, Tierney R, Vlahiotis A, Spitznagel EL and Piccirillo J: Changing epidemiology of Small cell lung cancer in the United States over the last 30 years: Analysis of the surveillance, epidemiologic, and end results database. J Clin Oncol 24(28): 4539-4544, 2006.

4 Lally BE, Geiger AM, Urbanic JJ, Butler JM, Wentworth S, Perry MC, Wilson LD, Horton JK, Detterbeck FC, Miller AA, Thomas CR Jr. and Blackstock AW: Trends in the outcomes for patients with limited stage small cell lung cancer: An analysis of the Surveillance, Epidemiology, and End Results database. Lung Cancer 64(2): 226-231, 2009.

5 Sekine I, Yamamoto N, Kunitoh H, Ohe Y, Tamura T, Kodama $\mathrm{T}$ and Saijo N: Treatment of small cell lung cancer in the elderly based on a critical literature review of clinical trials. Cancer Treat Rev 30(4): 59-368, 2004.

6 List AF, Hainsworth JD, Davis BW, Hande KR, Greco FA and Johnson DH: The syndrome of inappropriate secretion of antidiuretic hormone (SIADH) is small cell lung cancer. J Clin Oncol 4(8): 1191-1198, 1986.

7 Zarzecka M, Kubicki $\mathrm{P}$ and Kozielski J: Hyponatraemiaevaluation of prevalence in patients hospitalized in the pulmonary department and prognostic significance in lung cancer patients. Pneumonol Alergol Pol 82(1): 18-24, 2014.

8 Spasovski G, Vanholder R, Allolio B, Annane D, Ball S, Bichet D, Decaux G, Fenske W, Hoorn EJ, Ichai C, Joannidis M, Soupart A, Zietse R, Haller M, van der Veer S, Van Biesen W, Nagler E and Hyponatraemia Guideline Development Group: Clinical practice guideline on diagnosis and treatment of hyponatraemia. Eur J Endocrinol 170(3): G1-47, 2014.

9 Hansen O, Sørensen P and Hansen KH: The occurrence of hyponatremia in SCLC and the influence on prognosis. A retrospective study of 453 patients treated in a single institution in a 10-year period. Lung Cancer 68(1): 111-114, 2010.

10 Iyer AV, Krasnow SH, Dufour DR and Arcenas AS: Sodium wasting nephropathy caused by cisplatinum in a patient with small cell lung cancer. Clin Lung Cancer 5(3): 187-189, 2003.

11 Rosner $\mathrm{MH}$ and Dalkin AC: Electrolyte disorders associated with cancer. Adv Chronic Kidney Dis 21(1): 7-17, 2014. 
12 Ray P, Quantin X, Grenìer J and Pujol JL: Predictive factors of tumor response and prognostic factors of survival during lung cancer chemotherapy. Cancer Detect Prev 22(4): 293-304, 1998.

13 Cuesta $\mathrm{M}$ and Thompson C: The relevance of hyponatremia to peri-operative care of surgical patients. Surgeon 13(3): 163-169, 2015.

14 Sørensen JB, Andersen MK and Hansen HH: Syndrome of inappropriate secretion of antidiuretic hormone (SIADH) in malignant disease. J Intern Med 238(2): 97-110, 1995.

15 Littlewood TJ and Smith AP: Syndrome of inappropriate antidiuretic hormone secretion due to treatment of lung cancer with cisplatin. Thorax 39(8): 636-637, 1984.

16 Vanhees SL, Paridaens R and Vansteenkiste JF: Syndrome of inappropriate antidiuretic hormone associated with chemotherapyinduced tumour lysis in Small cell lung cancer: Case report and literature review. Ann Oncol 11(8): 1061-1065, 2000.

17 Hermes A, Waschki B and Reck M: Hyponatremia as prognostic factor in Small cell lung cancer-a retrospective single institution analysis. Respir Med 106(6): 900-904, 2012.

18 Lassen U, Osterlind K, Hansen M, Dombernowsky P, Bergman B and Hansen HH: Long-term survival in Small cell lung cancer: Posttreatment characteristics in patients surviving 5 to $18+$ years - An analysis of 1,714 consecutive patients. J Clin Oncol 13(5): 1215-1220, 1995.
19 Østerlind K and Andersen PK: Prognostic factors in small cell lung cancer: Multivariate model based on 778 patients treated with chemotherapy with or without irradiation. Cancer Res 46(8): 4189-4194, 1986.

20 Allan SG, Stewart ME, Love S, Cornbleet MA, Smyth JF and Leonard RC: Prognosis at presentation of small cell carcinoma of the lung. Eur J Cancer 26(6): 703-705, 1990.

21 Thajudeen B and Salahudeen AK: Role of tolvaptan in the management of hyponatremia in patients with lung and other cancers: Current data and future perspectives. Cancer Manag Res 8: 105-114, 2016.
Received May 19, 2017

Revised June 8, 2017

Accepted June 9, 2017 